\title{
Rehabilitation of visual functions in adult amblyopic patients with a virtual reality videogame: a case series
}

\author{
Carmen Jiménez-Rodríguez ${ }^{1}$. Lourdes Yélamos-Capel ${ }^{1}$ Patricia Salvestrini ${ }^{4}$. Cristian Pérez-Fernández ${ }^{1}$. \\ Fernando Sánchez-Santed ${ }^{1,3,6}\left[\right.$ [D $\cdot$ Francisco Nieto-Escámez ${ }^{1,2,5}$ (1)
}

Received: 24 June 2020 / Accepted: 4 November 2021 / Published online: 17 November 2021

(c) The Author(s) 2021

\begin{abstract}
Amblyopia or lazy eye is a dysfunction of the visual system that appears during childhood and traditionally has been considered untreatable in adults. Its main consequences are the loss of visual acuity and contrast sensitivity of the amblyopic eye and binocular vision impairments. During the last years videogames have been used as a therapeutic tool for amblyopia with the inconclusive results. The present work has assessed the effectiveness of a virtual reality videogame (AmbliOK®) in the neurorehabilitation of four adult clinical cases with anisometropic amblyopia. Visual acuity, contrast sensitivity, stereopsis and interocular suppression were assessed before, during, immediately after, one month and one year (in one patient) after the training. The intervention was conducted along four weeks $(10 \mathrm{~h})$ and yielded the variable results. In general, all patients showed an improvement in visual functions although not all ameliorated in the same way. Visual acuity measures improved in all patients, falling outside the amblyopia criterion at the end of the treatment. However, the improvement was not maintained one month later in two patients. Contrast sensitivity progressively improved for the amblyopic and the fellow eyes with all patients showing better results one month after the treatment. The patient assessed one year after still showed better results than in the baseline. Patients showing bad stereopsis in the baseline reached a performance considered normal one month and even one year after the treatment. The effectiveness of the treatment seems to be related to the characteristics of patients.
\end{abstract}

Keywords Amblyopia $\cdot$ Visual acuity $\cdot$ Contrast sensitivity $\cdot$ Stereopsis $\cdot$ Videogame $\cdot$ Virtual reality

\section{Introduction}

Francisco Nieto-Escámez

pnieto@ual.es

1 Department of Psychology, University of Almeria, Ctra. Sacramento S/N, 04120 Almería, Spain

2 Centre for Neurorehabilitation and Neuropsychological Assessment (CERNEP), Ctra. Sacramento S/N, 04120 Almeria, Spain

3 Centre for Health Research (CEINSA), Ctra. Sacramento S/N, 04120 Almería, Spain

4 QVision, Vithas Virgen del Mar Hospital, Ctra. Mami Km 1, 04120 Almería, Spain

5 NeuroDigital Technologies SL, Plaza Dalias S/N, 04007 Almeria, Spain

6 Institute for Child Neurohabilitation (INPAULA), C/Angel Gomez Fuentes 11, 04007 Almeria, Spain
Visual development takes place as result of a process of neurological maturation from birth to around the age of 8 years. As the first years of life are decisive in this process, any deficit regarding visual stimulation during critical periods of development interferes with the maturation of the visual brain, cutting off sensory processing and impairing perceptive mechanisms. This leads to a functional visual deficit that does not require injury or structural alterations in the eye named amblyopia (Capetillo et al. 2011).

Amblyopia or "lazy eye" has a prevalence of $1-5 \%$ (Bonaccorsi et al. 2014), and is commonly associated with uncorrected strabismus, anisometropia (high difference in refractive error between eyes), or cataract induced privation (Huang et al. 2011). Amblyopia mainly affects functions like visual acuity (VA), contrast sensitivity (CS), movement sensitivity or stereopsis (Bonaccorsi et al. 2014; Levi 2015). All these visual functions have an important 
role in daily activities (Hayhoe 2009). Another important characteristic of amblyopia is suppression. When one eye is turned, binocular normal vision is impaired producing diplopia or confusion. To avoid such sensory abnormalities, suppression works as an adaptative mechanism of the brain. Thus, the brain inhibits the image coming from the turned eye (Gil-Gibernau 1997; Merchante 2013; Kanski 2016).

Although the literature has traditionally focused on monocular deficits associated with the amblyopic eye, functional imbalance between the two eyes can lead to changes that also affect to the fellow eye, as well as binocular interactions between both eyes (Harrad and Hess, 1992; Harweth 1983; Mitchell et al. 2003; Smith and Trachtenberg, 2007). In clinical practice, there exist two classical treatments for amblyopia: refractive correction and occlusion of the fellow eye, and "penalization" treatment with atropine of the fellow eye. These treatments are considered as passive methods and both are employed during childhood, until 8 or 9 years old (Bonaccorsi et al. 2014). Not so many years ago, researchers were convinced that when critical periods ended it was impossible to modify the cortical representation of the weak eye (Bonaccorsi et al. 2014; Spolidoro et al. 2009). However, recent research has shown the possibility of brain plasticity in adults. Several studies have found that after monocular deprivation (a model of amblyopia), binocular activity was recovered with appropriate stimulation in adulthood (Berardi et al. 2003; Fagiolini and Hensch 2000; He et al. 2006, 2007; Spolidoro et al. 2009). Furthermore, anatomical and functional neural changes have been described (Bonaccorsi et al. 2014; Antonini et al. 1999; Mataga et al. 2004; Oray et al. 2004). Thereby, the possibility of treating amblyopia in adulthood has become a reality. With this premise and the idea of binocular stimulation as promoter of neuronal plasticity, dichoptic treatments have been used in the last years.

Dichoptic treatments work displaying the same background for both eyes, but the amblyopic eye also receives enriched stimuli. This treatment is based on the hypothesis that amblyopic patients have an intact binocular visual system, which becomes functionally monocular due to suppression mechanisms (Foss, 2017; Hess et al. 2011; Hess and Thompson, 2013; Levi et al. 2015). The advantage of a dichoptic treatment is the possibility of stimulating both monocular and binocular processes at the same time. However, amblyopia is more than a VA impairment. Other functions such as CS and stereopsis should be also trained. In order to improve CS and stereopsis, besides VA, perceptual learning has become part of treatment for amblyopia in the last years. Perceptual learning training consist of visual tasks that involve the discrimination of small stimuli features like position, orientation, shape, or texture (Bonaccorsi et al. 2014). Thus, perceptual learning training has demonstrated the possibility of recovering visual deficits (Polat 2009), or amblyopia (Levi and Li 2009; Li et al. 2007; Polat et al. 2004).

In the last years, as a result of technology development, many traditional instruments are being substituted by computer applications. In the case of amblyopia, its treatment has been favored with the use of virtual reality (VR) (Foss 2017). VR is a versatile tool that allows clinicians to adapt it to each specific disorder, particularly in the field of sensory rehabilitation and amblyopia treatment. Thus, VR has a number of advantages: (1) it is a low-cost procedure; (2) professionals can design the task within a wide range of adjustable parameters in monocular and binocular environments (Hernández-Rodríguez et al. 2020); (3) VR is compatible with high-precision functional imaging techniques, like functional magnetic resonance imaging (fMRI), which allows the clinicians to present multimodal stimuli with a high degree of validity and ecological control while recording changes in subject's brain (Coco-Martín et al. 2020); (4) The immersion feeling through head-mounted-devices (HMD) is another important characteristic of VR. HMDs introduce patients into a colorful, attractive and powerful environment to improve their performance and to go on through levels. This enhances motivation and increases patient's participation (Arlati et al. 2017; Coco-Martín et al. 2020; Hernández-Rodríguez et al. 2020).

VR allows to combine the characteristics of dichoptic strategies and perceptual learning. VR headsets are equipped with two separate lenses which allow to display different images to each eye, therefore, researchers can control which stimuli are presented to each eye separately, as well as the stimuli features such as the level of contrast. Although research has mainly focused on children, there are some studies with adults showing that the age of treatment is not a barrier for visual rehabilitation. Saraiva et al. (2018) using Google Cardboard reported that $98 \%$ of the patients improved after the treatment. In this work the authors developed an ocular therapy treatment procedure for strabismic patients based on eye movement exercises. Ziak et al. (2017) used the Oculus Rift DK2 headset to play a game called Diplopia Game (Vivid Vision, San Francisco, USA). Two games were employed: a space game in which patients were flying a spaceship through a set of rings and an Arkanoidtype game, both played in a 3D setting. Ziak et al. (2017) found that both VA and stereoacuity improved after training. Nevertheless, although some studies have used applications such as videogames, videoclips or smartphone applications to treat amblyopia, most of them have not been developed by eye care professionals or in a VR environment either (Paudel 2018).

In the present work we have used the Oculus Rift headset along with a videogame designed for amblyopia treatment called AmbliOK®. As a pilot study our goal has been to evaluate the effectiveness of this treatment in a group of 
adult anisometropic patients assessing the effect of the intervention across VA, SC, stereopsis and suppression measures. It is important to assess as many variables as possible, as the effect of the treatment can be different for each variable. All the variables have been assessed before, during, immediately after and at least one month after the treatment in order to detect immediate and long-time effects.

\section{Methods}

\subsection{Participants}

This study is composed by a series of four cases of anisometropic amblyopia. As criterion for amblyopia a minimum visual acuity difference of $0.2 \log$ MAR (ETDRS Sloan Optotypes) between the two eyes was considered. Exclusion criteria were the presence of eye pathologies, for instance ocular deviations and affections of the macula or the optic nerve, and the use of drugs that may interfere with the training or the assessment of visual functions. To recruit the participants several advertisements were posted on different social networks and across the campus. A total of 10 people contacted the research team and the appropriate evaluations were made. After these initial evaluations, only 4 met the inclusion criteria and the others were discarded for different reasons: 3 candidates did not meet the criteria for amblyopia, 1 was using anxiolytics and antidepressants, and 2 showed micro-strabismus.

Participant number one is a 63-year old woman with anisometropic amblyopia on the right eye. She received occlusion treatment with orthoptic patch at 8 years. She did not need refractive correction until she was diagnosed with presbyopia and astigmatism in adulthood.

Participant number two is an 18-year old man with anisometropic amblyopia on the right eye. He received occlusion treatment with orthoptic patch at 6 years.

Participant number three is a 25 -year old man with anisometropic amblyopia on the left eye. He did not receive any treatment in childhood. He abandoned the treatment with AmbliOK on the third week for reasons no related to the investigation.

Participant number four is a 42-year old woman with anisometropic amblyopia on the left eye. She received occlusion treatment with orthoptic patch at 7 years.

All patients received information about the research and signed the informed consent form.

\subsection{Initial assessment of ocular structure and function}

In order to discard possible ocular pathologies and establish a baseline for our study variables, different ophthalmic instruments and optometric tests were used:
Slit Lamp (Essilor SL) with SL 990 5X biomicroscopy® is to examine the anterior surface and bottom of the eye. With this test it is possible to detect diseases like glaucoma, cataract, or macular degeneration.

VRK-1900 Autoref-Keratometer ${ }^{\circledR}$ was used to measure the refraction value of each eye, interpupillary distance, pupil diameter, the angle axis and corneal curvature.

Cover-uncover test was used to discard any possible ocular deviation.

Optical coherence tomography was used to assess the state of the retina, the structure of the optic nerve and the macula.

\subsection{Evaluation of visual function variables}

Several optometric tests were conducted in order to establish a baseline for all the variables and the subsequent effects of the treatment. Visual functions were also evaluated weekly during the intervention phase, one month after (in all patients) and one year later (in one patient). Optometric tests were carried out at QVision S.L. Clinic of Almería.

The Worth four dot test provides qualitative information of binocular function, binocular rivalry (suppression) and flat fusion. The participants were required to wear red-green filters in order to control the input from different stimuli. The evaluation was made $400 \mathrm{~cm}$ from the screen, placed $160 \mathrm{~cm}$ from the floor. Stimuli were displayed on a VIC1900(P) monitor (ViewM Technology Ltd. Gyeonggi-do, South Korea).

The ETDRS Optotype (Precision Vision, Woodstock, Illinois, USA) is the most used instrument to determine static visual acuity. ETDRS provides a maximum value of $-0,3$ $\log$ MAR and a minimum value of 1,0 $\log$ MAR. 1,0 value shows poor vision, while a subject with appropriate visual function gets around $0.0 \log$ Mar values. Values below 0.0 means a better visual acuity than normal. Following the standard consideration for research purposes, $400 \mathrm{~cm}$ of distance was set from the chart to the subject. Illumination of the cabinet was fixed at standard criteria of $85 \mathrm{~cd} / \mathrm{m}^{2}$.

A CSV 1000 E (Vector Vision, Greenville, Ohio, USA) was used to evaluate contrast sensitivity with four spatial frequencies (3, 6, 12 and 18 cycles per degree) and eight contrast levels. Low spatial frequencies assess the sensitivity of very large objects. On the contrary, high spatial frequencies assess the sensitivity of very small objects. The chart was placed $160 \mathrm{~cm}$ from the floor and the participants were set at $250 \mathrm{~cm}$ from the chart.

The titmus test or fly test with Lea symbols P/N 1000 (Vision Assessment Corporation, Elk Grove Village, Illinois, USA) was used to measure gross and fine stereopsis. The graded circle test was used to assess fine stereopsis in the range from 400 to 20 s/arc. 


\subsection{Hardware and videogame for visual treatment}

We used a PC (Intel® Core ${ }^{\mathrm{TM}}$ i7-2600, 16 GB RAM, Nvidia GeForce 970GT 8 GB) and the Oculus Rift DK2 headset (Oculus VR LLC, Irvine, California, USA) for displaying the game.

AmbliOK $®$ (NeuroDigital Technologies SL, Almeria, Spain) was used as rehabilitation software. AmbliOK is based on the classic videogame Arkanoid, with a Halloween inspired aesthetic. The videogame displayed a two-dimensional scenario in which different objects were included: a ball moving across the screen, a pad at the bottom of the screen, which the participant could move horizontally by using the keyboard left and right arrow keys in order to intercept the ball. Halloween pumpkins were displayed in the upper half of the screen which blew up when the ball impacted them. The game goal was to destroy all the pumpkins while avoiding the ball fall through the bottom of the screen. All elements were displayed binocularly except the ball which was displayed to the amblyopic eye only. AmbliOK ${ }^{\circledR}$ has four levels of difficulty (incrementing the ball's speed). During the four weeks of treatment the four levels were used (Fig. 1).

\subsection{Procedures}

This investigation was approved by the Bioethical Committee of the University of Almería for human research. It has been done according with the Spanish Law 14/2007 for Biomedical Research and has been conducted according to the tenets of the Helsinki's Declaration (2013). All participants signed an informed consent form and received an informative sheet explaining study's characteristics. All patients participated voluntarily in this study.
Several candidates were initially evaluated by an optometrist at QVision SL Clinic facilities. However, only four participants meet the criteria to participate in the study.

Initial optometric assessments were used to establish a baseline for VA, CS, stereopsis, and suppression. Before each training session, the Oculus Rift DK2 was adapted and calibrated for each patient.

The intervention phase with the videogame was carried out for four weeks, with five sessions of $30 \mathrm{~min}$, once per day from Monday to Friday. This protocol was based on previous pilot studies that showed it was effective and bearable. The total training time during the four weeks was $10 \mathrm{~h}$. Every session, the participant was sat in front of the computer in a quiet room wearing the VR headset. As described in 2.4, the participants had to track the ball displayed to the amblyopic eye and avoid it disappeared through the bottom of the screen. In such case a life was lost. (Participants started the game with three lives.) Every week a new level of difficulty was used increasing the ball's speed. The rationale was to keep the interest of the participant as the game was very easy to play. A scoreboard was displayed all the time in order to motivate the participants. Each time all the pumpkins were destroyed a new screen filled with pumpkins was automatically displayed and the participant got an extra life. In case the participant lost all the lives the game was restarted manually.

The assessment of all visual variables was done at the end of every training's week. We did a follow up assessment one month after the intervention. Patients did not receive any treatment during this month. The participant number one also did a follow up assessment one year after the treatment had ended.
Fig. 1 Screenshot showing the game AmbliOK configured to train the right eye. The background was the same for both eyes but only the image corresponding to the right eye displayed the ball (upper part of the game screen) that participants had to track, preventing it fall through the bottom

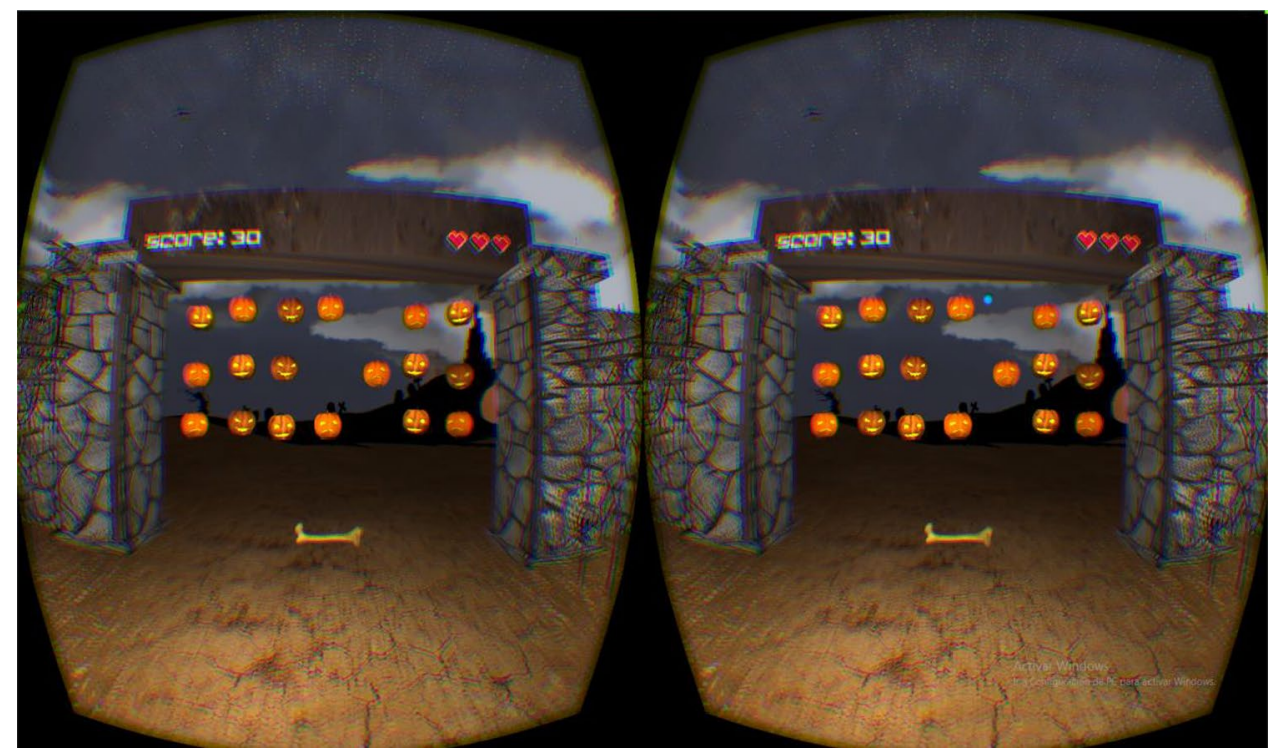




\section{Results}

\subsection{Participant 1}

Participant 1 showed a VA difference of $0.22 \log$ MAR between the fellow eye (left) and amblyopic eye (right) in her baseline assessment. Initial CS was poor with only 2 stimuli (out of 8 ) detected in low frequency range $(3 \mathrm{c} / \mathrm{g}$ ). Stimuli of higher frequencies were not detected. Stereopsis scoring showed a value of 160 arc of second (160"). See Figs. 2, 3 and 4 baseline scores.

During the intervention phase, amblyopic eye VA improved significantly and, from the second week, the patient overcame the criterion of amblyopia ( $0.2 \log$ MAR difference between two eyes). This improvement disappeared one month after and the participant fell back into the criterion of amblyopia. Binocular VA fluctuated during the intervention but fell back into the criterion of amblyopia one month after. One year later VA remained as in the baseline (Fig. 2).

CS showed a progressive improvement during the intervention phase. The patient was able to discriminate with her amblyopic eye 5 or 6 items (out of 8 ) in all the frequencies range at the end of the treatment. The improvement remained one month later. The fellow eye also showed a progressive improvement in all the frequencies. This improvement also remained a month after the training's end. One year later, (a)

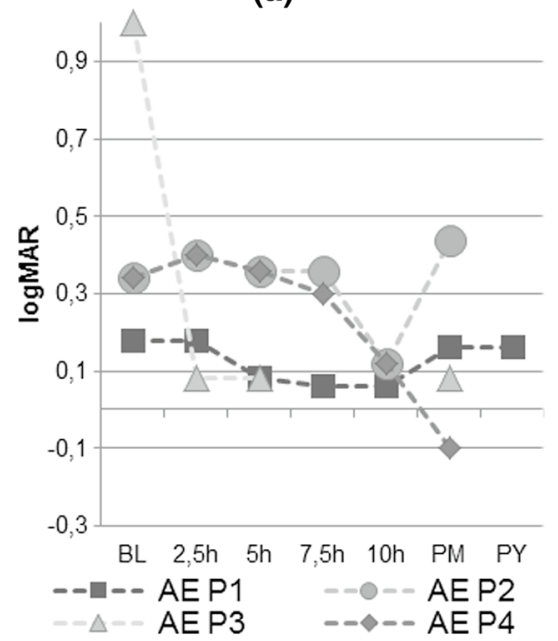

(b)

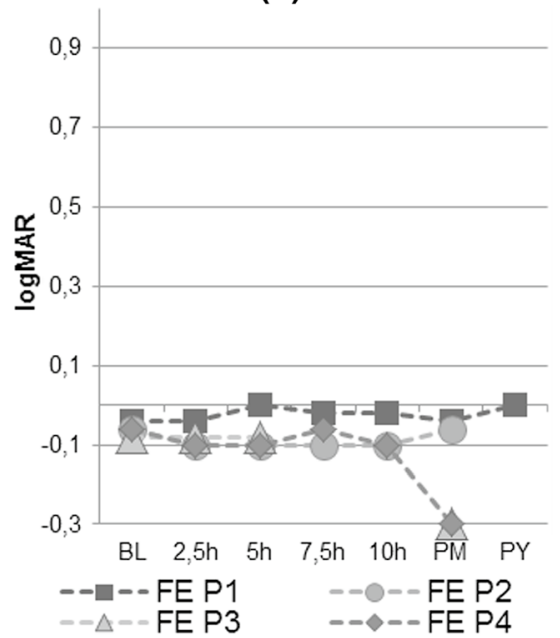

(c)

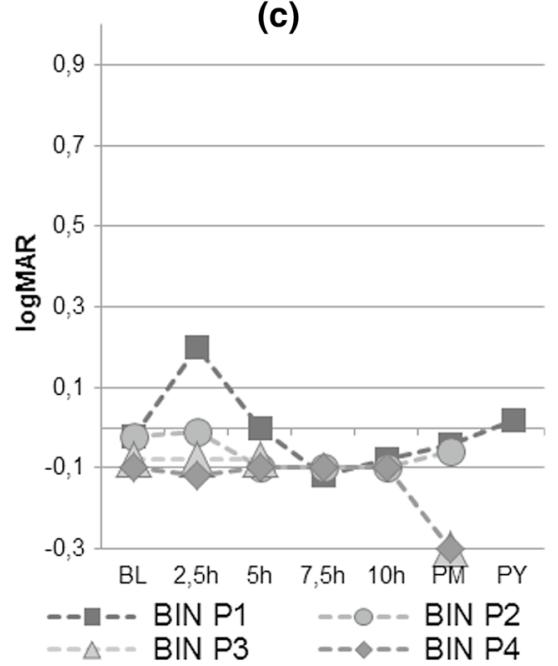

Fig. 2 Evolution of visual acuity along the training, 1 month and 1 year after for the amblyopic eye (a), the fellow eye (b) and binocular (c). BL: base line; PM: 1 month follow-up; PY: 1 year follow-up; AE: amblyopic eye; FE: fellow eye; BIN: binocular; P1-P4: participants

(a)

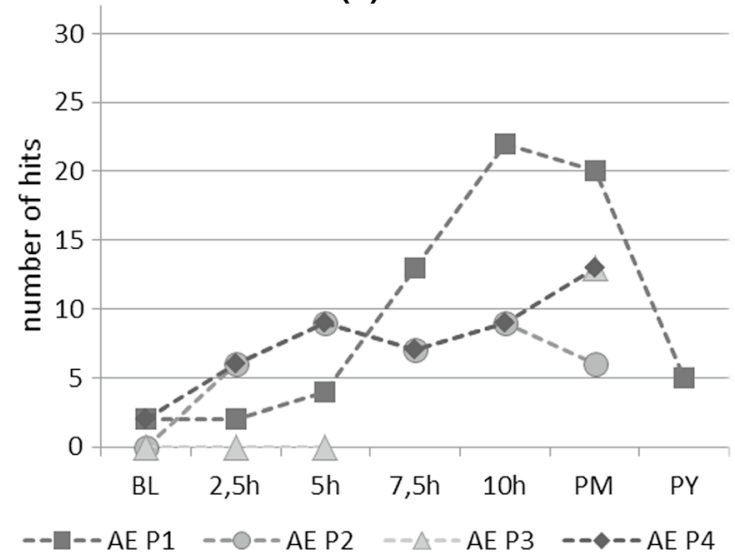

Fig. 3 Evolution of sensibility evolution (scores from all the frequencies have been aggregated) along the training, 1 month and 1 year after for the amblyopic eye (a) and the fellow eye (b). BL: base line; (b)

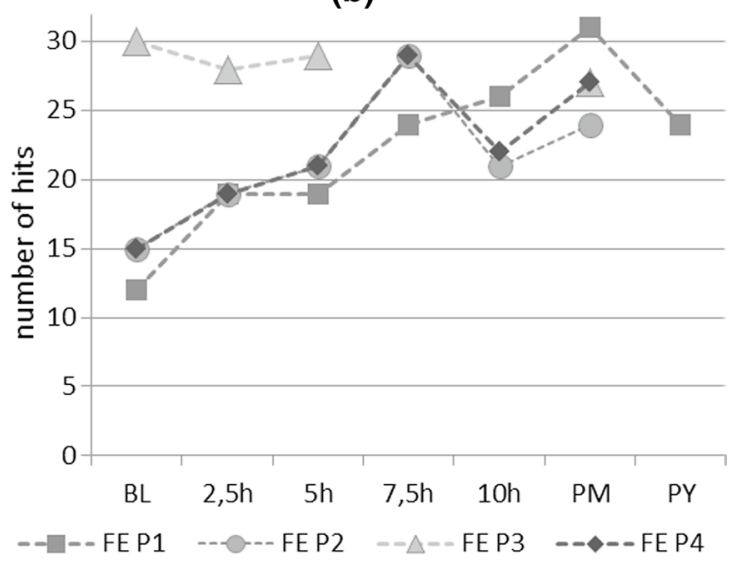

PM: 1 month follow-up; PY: 1 year follow-up; AE: amblyopic eye; FE: fellow eye; BIN: binocular; P1-P4: participants 


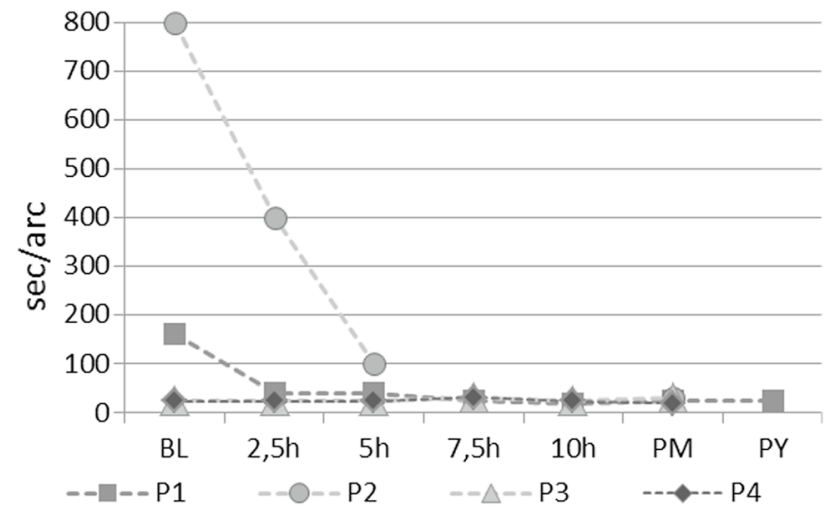

Fig. 4 Evolution of stereopsis along the training, 1 month and 1 year after. BL: base line; PM: 1 month follow-up; PY: 1 year follow-up; AE: amblyopic eye; FE: fellow eye; BIN: binocular; P1-P4: participants

the amblyopic eye showed worse scores than in the previous evaluation (a month after the intervention) but better than in the baseline. The fellow eye scoring maintained its improvement (Fig. 3).

Stereopsis improved from the first week and progressively achieved normative scores. Such improvement remained one month and one year after the intervention (Fig. 4).

Lastly, participant 1 showed no suppression during the whole study.

\subsection{Participant 2}

Participant 2 showed a VA difference of $0.4 \log$ MAR between the fellow eye (left) and amblyopic eye (right) in the base line score. CS scoring was poor as he only detected 2 out of 8 items in the low frequency range. The fellow eye showed a good CS for lower frequencies but not for higher frequencies. Stereopsis scored above normal vision (25"). However, the participant showed suppression of the right eye (amblyopic eye). This disappeared after 3 weeks of training and it was maintained one month later. See Figs. 2, 3 and 4 baseline scores.

VA remained stable during the treatment showing an improvement in the last week of intervention (amblyopia criterion disappeared). One month later this improvement disappeared in the follow up assessment falling under the baseline score (Fig. 2).

Participant 2 showed a progressive improvement during the intervention phase for CS at $3 \mathrm{c} / \mathrm{g}$ and $6 \mathrm{c} / \mathrm{g}$ frequencies. However, only the improvement in the $3 \mathrm{c} / \mathrm{g}$ frequency remained one month later. No improvements were observed for higher frequencies. The Fellow eye results were stable during the intervention with a slight evolution for $12 \mathrm{c} / \mathrm{g}$ and $18 \mathrm{c} / \mathrm{g}$ frequencies.
Stereopsis scores remained stable during the whole study and comparable to normal vision (Fig. 4).

\subsection{Participant 3}

Participant 3 showed a VA difference of $1.08 \log$ MAR between both eyes in the baseline. Regarding CS, this participant was unable to discriminate any item at any frequency, and showed extremely poor stereopsis $\left(800^{\prime \prime}\right)$ in the baseline assessment. By contrast, fellow eye CS was really good as the participant could discriminate almost all low frequencies and all the high frequencies. This participant showed suppression of the left eye for the whole study duration. This participant left the investigation after two weeks for reasons beyond the research. See Figs. 2, 3 and 4 baseline scores.

VA improved notably after only one training week. This achievement remained during the second week and improved even more one month later reaching -0.08 $\log$ MAR for the amblyopic eye and $-0.3 \log$ MAR for the fellow eye (Fig. 2).

CS did not show any change during the training but one month later there was a remarkable improvement as this participant could discriminate 6 out of 8 items for $3 \mathrm{c} / \mathrm{g}, 3$ out of 8 items for $6 \mathrm{c} / \mathrm{g}, 1$ out of 8 items for $12 \mathrm{c} / \mathrm{g}$, and 3 out of 8 items for $18 \mathrm{c} / \mathrm{g}$ frequencies. In the baseline, this participant could not discriminate any item. Fellow eye scores were stable during the training period and similar to baseline results (Fig. 3).

Stereopsis also improved notably from $800^{\prime \prime}$ in the baseline to $100^{\prime \prime}$ after two weeks of training. One month later, a normal stereopsis score was observed (Fig. 4).

\subsection{Participant 4}

Participant 4 showed a VA difference of $0.4 \log$ MAR between both eyes in the baseline. CS was poor as she only could discriminate 2 out of 8 items in the lower frequency range. The fellow eye showed better performance for low frequencies although did not discriminate all the items. Stereopsis was comparable to normal vision. This participant did not show suppression. See Figs. 2, 3 and 4 baseline scores.

Regarding VA, it improved notably, overcoming the criterion for amblyopia at the end of training. One month later, amblyopic and fellow eyes and binocular VA improved over the last evaluation score at the end of the last training week (Fig. 2).

CS improved progressively during the training and one month later, showing even better scores for higher frequencies (Fig. 3).

Stereopsis scoring was comparable to normal vision in the baseline and during all the evaluations (Fig. 4). 


\section{Discussion}

In the present research we have tried to verify the effectiveness of virtual reality for the treatment of anisometropic amblyopia. Thus, we have evaluated the effects of dichoptic training using the video game AmbliOK for a maximum of $10 \mathrm{~h}$ (using Oculus Rift DK2 glasses) on VA, CS, stereopsis and suppression of the amblyopic eye in a series of four adult patients. Overall, although there is a significant variability across patients, an improvement in some variables has been observed during the training phase with the videogame, in the subsequent evaluations one month after the end of the training, and even one year later (in the only case where the participant could be evaluated). We observed that, even though the four participants were exposed to the same training protocol (one of the patients only completed 2 weeks of training), the benefits of the treatment varied across the four patients.

The VA results are generally encouraging in all patients. The 4 patients overcame the amblyopia criterion during the training with AmbliOK. These results agree with other works that have studied the effects of VR and videogames in visual rehabilitation (Cleary et al. 2009; Herbison et al. 2013; Waddingham et al. 2006; Ziak et al. 2017). Nevertheless, we should mention a recent study by Gao et al. (2018) who reported no significant improvements for VR treatments compared to placebo. Methodological differences, such as the design of the game, the fact that it was applied at home and not in a clinical setting or the followup of the treatment may explain the differences. Gao et al. (2018) point out that as their treatment was applied at home, it could had caused the participants did not pay the required attention to the task or did not perform the treatment during the prescribed hours. Nevertheless, patients' improvements could be seen in different variables (as seen in our results) without a global significant improvement when each individual variable was analyzed across the group. Thus, some patients improved their VA, others their CS, others their stereopsis or a combination of different variables.

In the assessment carried out one month after the intervention (and one year later for participant 1) it was observed that participants 3 and 4 maintained the VA improvement for the amblyopic eye. In addition, participant 4 obtained better scores in the month followup compared to the evaluation carried out immediately after the treatment. This result is in line with previous research showing perceptual learning training produced an improvement in VA and CS measures which were maintained some months after the end of the training (Barollo et al. 2017; Clearly et al. 2009; Mansouri et al. 2014; Moret et al. 2018; Ziak et al. 2017). Barollo et al. (2017) defined the maintenance of the improvement as "retention of the effect of the perceptual learning". According to these authors, a long-term maintenance of the effects of the training is caused by the presence of a latent phase that lasts several hours. This phase would reflect a consolidation of the trained mechanisms and during this time daily life activities involving VA and CS functions would work as a continuous training. These results indicate that medium- and long-term assessment of the improvements must be included in future investigations. As Ziak et al. (2017), who only completed the medium-term follow-up for 3 patients, we have not yet done a full long-term follow-up (after one year) for all patients.

Additionally, our participants also showed an improvement in their fellow eye VA during and/or at the end of the training, and one month later. The maintenance of this improvement is explained in the same way as for the amblyopic eye. According to Meier and Giaschi (2017), although the fellow eye shows better performance than the amblyopic eye, its performance is not equivalent to a control healthy eye. Following these authors, the pattern of atypical development suffered by the visual system of amblyopic people, both monocularly and binocularly, would also cause an anomalous development in their fellow eye visual pathways. Therefore, as shown by Barollo et al. (2017), Zhou et al. (2006), Huang et al. (2008), Li et al. (2007) or Moret et al. (2018), the fellow eye would benefit from an interocular transfer resulting from the stimulation of the amblyopic eye. This stimulation would create new connections leading to changes at binocular cortical level and the improvement observed in the fellow pathway. This would explain the improvements observed after patching the fellow eye (Barollo et al. 2017; Li et al. 2007; Moret et al. 2018). A similar explanation was offered by Hess et al. (2011) on basis to the strengthening of the interactions between binocular neurons that previously had been suppressed.

Regarding the evolution of CS in the amblyopic eye we have observed a progressive improvement throughout the training that was maintained for lower frequencies one month after the training although in participant 1 disappeared one year later. Moreover, we have also observed an improvement in the fellow eye which was not exposed to the stimulation condition of the videogame. It can be argued that the video game also produces a certain training effect on the non-amblyopic eye. Thus, while the fundamental element of the game is to track the trajectory and movement of the ball (only presented to the amblyopic eye), the fellow eye was also exposed to the rest of the scene and to the movement of the pad that the player moved to intercept the ball. Additionally, we must point that the VR videogame, unlike monocular stimulation procedures, uses a binocular training strategy. As discussed above, amblyopia also affects the development of fellow eye pathways (Meier and Giaschi, 
2017). Varadharajan and Hussaindeen (2012) showed that fellow eye's VA in children with amblyopia is worse when compared to non-amblyopic control eyes. Consequently, dichoptic stimulation would also improve CS of the fellow eye.

With regard to the physiological bases of the fellow eye improvement, it could be associated with changes in the functioning of binocular neurons that might influence vision in an up-down way. Meier and Giaschi (2017) argue that the deficits of the fellow eye are usually linked to specific extrastriate visual cortex that include a large number of binocular neurons. These binocular mechanisms can be independent of the particular eye from which they receive the information, producing phenomena like interocular transfer. Very few works have paid attention to the improvement in the fellow eye in amblyopic subjects after training with perceptual learning or other techniques. With regard to CS, Moret et al. (2018) argue that its improvement is due to a weakening of inhibitory lateral interactions between V1 neurons for specific spatial orientations and frequencies (Polat 1999; Polat et al. 2004), with a subsequent decrease of interocular suppression (Harrad 1996). The explanation of Hess et al. (2011) on the strengthening interaction between binocular neurons would be also applicable to the case of CS.

In addition, the improvement in CS observed one month after the end of the intervention in some participants is striking. It would indicate that regardless the visual training procedure, once the process of cortical plasticity underlying the improvement has begun, this mechanism remains active even after the training sessions have been completed. There could be a generalization of the response as shown by various studies (Casco et al. 2014; Huang et al. 2008; Polat et al. 2004; Zhou et al. 2006), and it could be possible that, as with VA, the stimulation of daily activities continues the rehabilitation process (Barollo et al. 2017).

Regarding stereopsis, the improvement in binocular performance is a main goal of dichoptic treatments. It should be noted that participants 1 and 3 showed a remarkable improvement in stereopsis, while participants 2 and 4 had already a good pre-training stereopsis that remained throughout the training. Stereopsis improvement is in line with the results of Ziak et al. (2017) for stereoacuity. These authors showed a significant improvement in stereoacuity even in patients who had a non-measurable stereopsis (above $800 ")$ in the baseline assessment. The same applies to one of our patients. In general, the improvement in the stereopsis of our patients is consistent with the data of Vedamurthy et al. (2016) or Ding et al. (2011) who also reported a great recovery in stereopsis after perceptual learning training. We should point out that, apart from the study by Vedamurthy et al. (2016) and Ziak et al. (2017), we do not know other studies using VR for stereopsis treatment in amblyopic patients. Stereopsis, as Levi et al. (2015) point out, is not a single global function but is broken down into different mechanisms and depends on binocular visual acuity and normal eye alignment (Von Noorden and Campos 2002). The multifactorial nature of stereopsis is reflected in the variability of patients' etiology. This would be the case for those who are considered stereo-blind but show residual stereoscopic functions (Mccoll et al. 2000; Harris and Sumnall 2000).

The precise mechanism by which anisometropia causes a decrease in stereopsis is not clear. As mentioned above, it has been suggested that foveal suppression in the defocused eye is the cause of the decrease in stereopsis (Nabie et al. 2019). McKee et al. (2003) propose that subjects with amblyopia and normal ocular alignment could have experienced degraded but concordant visual inputs, at least at low spatial frequencies, and therefore binocular vision could have developed. Fawcett and Birch (2003) point out that binocularity can be experienced with low levels of anisometropia, and Robaei et al. (2007) reported that $78.6 \%$ of children with anisometropia reached normal levels of stereopsis compared to $98.9 \%$ of children without anisometropia. Furthermore, previous studies (Julesz and Miller 1975; Ogle and Wakefield 1967) have informed conditions in which interocular suppression and stereopsis can be dissociated. What does seem clear is that, as Vedamurthy et al. (2016) and Ziak et al. (2017) point out, the high level of motivation associated with RV videogames results in better outcomes and could contribute to the beginning of neuronal plasticity. This would be the case of AmbliOK, whose playful component provides extra motivation for the patient.

Taking suppression as a possible cause of amblyopia, Li et al. (2013) hypothesized that alleviating suppression through the presentation of dichoptic stimuli results in a higher level of plasticity than forcing the use of the amblyopic eye. Suppression is a mechanism that prevents the amblyopic brain learns to see again. Nonetheless, the role, origin, and nature of suppression in amblyopia have not always been viewed this way (Barrett et al. 2012; Holopigian et al. 1986). Thus, it has been suggested that suppression can take different forms in anisometropia and strabismus: passive in anisometropia (where the image of the amblyopic eye is blurred) but active in strabismus to avoid diplopia (Levi et al. 2015). According to the last idea, Chen et al (2020) observed a substantial improvement in VA although interocular suppression did not disappear. It agrees with our results for participants 2 and 3, who showed good VA and excellent stereopsis with suppression of the amblyopic eye. Participant's 2 results suggest that training with AmbliOK could help to remove the interocular suppression. Probably, this would depend on a dose-response rate. (Table 1).

We would like to point out that the training duration in our study varies from Ziak et al. (2017). Currently there is no consensus on the optimal duration of training but, as these 
Table 1 Interocular Suppression progress in the 4 participants assessed with the Worth 4-dot Test

\begin{tabular}{llllllll}
\hline Participant & Base line & 1st week 2.5 h & 2nd week 5 h & 3rd week 7.5 h & 4th week 10 h & Post month & Post year \\
\hline 1 & 4 lights (NS) & 4 lights (NS) & 4 lights (NS) & 4 lights (NS) & 4 lights (NS) & 4 lights (NS) & 4 lights (NS) \\
2 & 3 lights (RS) & 3 lights (RS) & 3 lights (RS) & 4 lights (NS) & 4 lights (NS) & 4 lights (NS) & - \\
3 & 2 lights (LS) & 2 lights (LS) & 2 lights (LS) & - & - & 4 lights (NS) & - \\
4 & 4 lights (NS) & 4 lights (NS) & 4 lights (NS) & 4 lights (NS) & 4 lights (NS) & 4 lights (NS) & - \\
\hline
\end{tabular}

$N S$ No suppression, $R S$ Suppression of the right eye, $L S$ Suppression of the left eye

authors point out, it seems that at least 8 sessions ( $320 \mathrm{~min}$ ) are required to obtain good results. This "dose-response" ratio seems to indicate that more sessions mean a greater effect (Hamm et al. 2018), although it can be influenced by characteristics inherent to each patient. In Hamm et al. (2018), a longer play time produced additional improvements in VA and CS in 2 out of 3 groups of subjects with amblyopia by early bilateral deprivation. In addition, like us, they also obtained quite diverse results among groups and variables. The possible explanation given to this variability is the influence of variables such as the age and the origin of amblyopia, but also previous treatments. Consequently, previous interventions could have positive or negative effects on a subsequent binocular treatment. In future research it will be necessary to adapt the treatment to the characteristics of each patient by taking into account not only the training protocol but also the game features. As not every patient shows the same deficits it would be helpful that game features and mechanics were properly fitted to the needs of each treatment. In our case, AmbliOk was designed to make the amblyopic eye the main responsible for the gameplay (tracking the ball trajectory on the screen). In a similar way, Ziak et al. (2017) and more recently Halicka et al (2020) used Diplopia Game (Vivid Vision, USA) which displayed different stimuli to the amblyopic and the fellow eye, although in this case only the central part of the scene was different, in contrast to AmbliOk where the amblyopic eye must track the position of the ball across the whole screen. Another dichoptic strategy has been employed by Gambacorta et al. (2018), who designed a videogame that displayed the same scene to both eyes, although the contrast level between the amblyopic and the fellow eye was different. Although all these studies rely on the use of dichoptic stimulation, different procedures have been employed, resulting in an overall positive outcome although with significant variability among patients. Nevertheless, it is difficult to know if differences in game features have an impact in the results obtained by different authors. It can be worthy to understand which game characteristics are linked to particular outcomes. Further research should test which are the most efficient game features to train each visual variable taking also into account the characteristics of each patient.
A similar reasoning must be done regarding the technology used for visual training. The Oculus DK2 headset employed in the present study, as well as by Ziak et al. (2017) and Halicka et al. (2020), was released in 2014 and includes one single OLED display with a resolution of $960 \times 1080$ pixels per eye, and a field of view of $94^{\circ}$ horizontal and $99^{\circ}$ vertical. These specs have been already surpassed by later devices. We cannot discard that visual training could yield better results by using another HMD with superior specs. This could also depend on the characteristics of the patient, as in a previous pilot study using an older device (Oculus DK1) we observed a complete recovery of VA and stereopsis in a patient (after three weeks of training), which remained at least for 3 months after the training (unpublished results).

As a main conclusion of the preliminary results obtained with the videogame AmbliOK we can affirm that it is a valuable tool for training the visual functions affected in adults with anisometropic amblyopia. Moreover, the procedure has shown to be effective in some cases after a relatively brief intervention. This is particularly relevant as adults are usually considered an untreatable population. Thus, traditional therapies like eye occlusion are not prescribed beyond 8-9 years old. Furthermore, as discussed above, in the last years some authors have shown several strategies that are effective for visual rehabilitation in amblyopia. However, these works rely on the assessment of a reduced number of visual variables, usually no more than two. As we have shown, some patients show improvements in some visual variables whereas others show improvements in different ones. Therefore, in order to know if a treatment is effective it is necessary to conduct a comprehensive assessment of visual function, including at least VA, CS, stereopsis and suppression.

As our work is a series of cases, the small sample size appears as the main limitation. Moreover, the characteristics of each subject imply a significant degree of variability that greatly influences the results. Thus, the short number of participants impedes an analysis of the results according to their clinical characteristics, so it is not possible to know the reason for the differences between participants and variables. Nevertheless, as Ziak et al. (2017) and Foss et al. (2017) have reported dichoptic and videogame treatments involve 
a percentage of patients who do not show improvements. Another main limitation is the absence of a long term follow up in the majority of participants, as only one patient was available for the follow up one year after the intervention. In the coming future, we want to provide further evidence on the usefulness of our paradigm with AmbliOK carrying out a randomized controlled trial (RCT) with a wider sample. Finally, a more advanced HMD device should be used in further research.

Author's contribution All authors contributed to the study conception and design. Material preparation, data collection and analysis were performed by Carmen Jimenez-Rodriguez, Lourdes Yelamos-Capel and Patrizia Salvestrini. The first draft of the manuscript was written by Carmen Jimenez-Rodriguez and Lourdes Yelamos-Capel, and all authors commented on previous versions of the manuscript.

Funding Open Access funding provided thanks to the CRUE-CSIC agreement with Springer Nature. This research was supported by research grants PS09-01,163 from FIS, and PSI2014-55,785-C2-1-R from MINECO and EU FEDER funds. Ms. C.J.R is funded by an ONCE scholarship "Oportunidad al Talento" for PhD students.

\section{Declarations}

Conflicts of interest F.N.E. holds equity and has the role of Chief Scientific Officer at NeuroDigital Technologies S.L. The remaining authors declare no conflict of interest.

Ethics approval This study has been approved by the Ethics Committee for Human Research of the University of Almería. Moreover, it has been done following the ethical principles of the Declaration of Helsinki (2013) and the resolution 466/2012 of the National Health Council.

Consent to participate All participants were informed about the research characteristics and gave their informed consent before participation.

Consent for publication All authors read and approved the final manuscript.

Open Access This article is licensed under a Creative Commons Attribution 4.0 International License, which permits use, sharing, adaptation, distribution and reproduction in any medium or format, as long as you give appropriate credit to the original author(s) and the source, provide a link to the Creative Commons licence, and indicate if changes were made. The images or other third party material in this article are included in the article's Creative Commons licence, unless indicated otherwise in a credit line to the material. If material is not included in the article's Creative Commons licence and your intended use is not permitted by statutory regulation or exceeds the permitted use, you will need to obtain permission directly from the copyright holder. To view a copy of this licence, visit http://creativecommons.org/licenses/by/4.0/.

\section{References}

Antonini A, Fagiolini M, Stryker MP (1999) Anatomical correlates of functional plasticity in mouse visual cortex. J Neurosci 19(11):4388-4406. https://doi.org/10.1523/JNEUROSCI.19-1104388.1999

Arlati S, Zangiacomi A, Greci L, Di Santo SG, Franchini F, Sacco M (2017) Virtual enviroments for cognitive and physical training in ederly with mild cognitive impairment: a pilot study. In: De Paolis L, Bourdot P, Mongelli A (eds) Augmented Reality, Virtual Reality, and Computer Graphics. AVR 2017. Lecture Notes in Computer Science, vol 10325. Springer, Cham. https://doi.org/ 10.1007/978-3-319-60928-7_8

Barollo M, Contemori G, Battaglini L, Pavan A, Casco C (2017) Perceptual learning improves contrast sensitivity, visual acuity, and foveal crowding in amblyopia. Restor Neurol Neurosci 35(5):483496. https://doi.org/10.3233/rnn-170731

Barrett B, Panesar G, Scally A, Pacey I (2012) A Limited Role for suppression in the central field of individuals with strabismic amblyopia. PLoS ONE 7(5):e36611. https://doi.org/10.1371/journ al.pone. 0036611

Berardi N, Pizzorusso T, Ratto GM, Maffei L (2003) Molecular basis of plasticity in the visual cortex. Trends Neurosci 26(7):369-378. https://doi.org/10.1016/S0166-2236(03)00168-1

Bonaccorsi J, Berardi N, Sale A (2014) Treatment of amblyopia in the adult: insights from a new rodent model of visual perceptual learning. Front Neural Circ 8:82. https://doi.org/10.3389/fncir. 2014.00082

Capetillo OB, Triana-Casado I, Martínez-Legón Z, Roche-Caso S, Broche-Hernández A (2011) Frequency of amblyopia in school children. Rev Cubana Pediatr 83(4):372-381

Casco C, Guzzon D, Moise M, Vecchies A, Testa T, Pavan A (2014) Specificity and generalization of perceptual learning in low myopia. Restor Neurol Neurosci 32(5):639-653. https://doi.org/10. 3233/rnn140389

Chen Y, He Z, Mao Y, Chen H, Zhou J, Hess R (2020) Patching and suppression in amblyopia: One mechanism or two? Front Neurosci 13:1364. https://doi.org/10.3389/fnins.2019.01364

Cleary M, Moody AD, Buchanan A, Stewart H, Dutton GN (2009) Assessment of a computer-based treatment for older amblyopes: the Glasgow Pilot Study. Eye (lond) 23(1):124-131. https://doi. org/10.1038/sj.eye.6702977

Coco-Martín MB, Piñero D, Leal-Vega L, Hernández-Rodríguez CJ, Adiego J, Molina-Martín A, de Fez D, Arenillas JF (2020) The potential of virtual reality for inducing neuroplasticity in children with amblyopia. J Opthalmol. https://doi.org/10.1155/2020/ 7067846

Ding J, Levi D (2011) Recovery of stereopsis through perceptual learning in human adults with abnormal binocular vision. Proc Natl Acad Sci 108(37):E733-E741. https://doi.org/10.1073/pnas.11051 83108

Fagiolini M, Hensch TK (2000) Inhibitory threshold for critical-period activation in primary visual cortex. Nature 404(6774):183-186. https://doi.org/10.1038/35004582

Fawcett S, Birch E (2003) Validity of the Titmus and Randot circles tasks in children with known binocular vision disorders. J AAPOS 7(5):333-338. https://doi.org/10.1016/s1091-8531(03)00170-8

Foss A (2017) Use of video games for the treatment of amblyopia. Curr Opin Opthalmol 28(3):276-281. https://doi.org/10.1097/icu. 0000000000000358

Gambacorta C, Nahum M, Vedamurthy I, Bayliss J, Jordan J, Bavelier D, Levi DM (2018) An action video game for the treatment of amblyopia in children: a feasibility study. Vis Res 148:1-14. https://doi.org/10.1016/j.visres.2018.04.005 
Gao TY, Guo CX, Babu RJ et al (2018) Effectiveness of a binocular video game vs placebo video game for improving visual functions in older children, teenagers, and adults with amblyopia: a randomized clinical trial. JAMA Ophthalmol 136(2):172-181. https:// doi.org/10.1001/jamaophthalmol.2017.6090

Gil-Gibernau JJ, Cavero-Roig L, García-Arumí J, Güell JL, PascualCuch R, Rodrigo-Guzmán J (1997) Tratado de oftalmología pediátrica. Scriba, Barcelona

Halicka J, Sahatqija E, Krasnansky M, Kapitanova K, Fedorova M, Ziak P (2020) Visual training in virtual reality in adult patients with anisometropic amblyopi. Cesk Slov Oftalmol 76(1):24-28

Hamm L, Chen Z, Li J, Dai S, Black J, Yuan J et al (2018) Contrastbalanced binocular treatment in children with deprivation amblyopia. Clin Exp Optom 101(4):541-552. https://doi.org/10.1111/ cxo. 12630

Harrad R (1996) Psychophysics of suppression. Eye 10(2):270-273. https://doi.org/10.1038/eye.1996.57

Harrad R, Hess R (1992) Binocular integration of contrast information in amblyopia. Vision Res 32(11):2135-2150. https://doi. org/10.1016/0042-6989(92)90075-t

Harris J, Sumnall J (2000) Binocular three-dimensional motion detection: contributions of lateral motion and stereomotion. J Opt Soc Am A Opt Image Sci vis 17(4):687-696. https://doi. org/10.1364/josaa.17.000687

Harwerth R, Levi D (1983) Psychophysical studies of the binocular processes of amblyopes. Optom vis Sci 60(6):454-463. https:// doi.org/10.1097/00006324-198306000-00006

Hayhoe M, Gillam B, Chajka K, Vecellio E (2009) The role of binocular vision in walking. Vis Neurosci 26(1):73-80. https://doi. org/10.1017/S0952523808080838

He HY, Hodos W, Quinlan EM (2006) Visual deprivation reactivates rapid ocular dominance plasticity in adult visual cortex. J Neurosci 26(11):2951-2955. https://doi.org/10.1523/JNEUR OSCI.5554-05.2006

He HY, Ray B, Dennis K, Quinlan EM (2007) Experience-dependent recovery of vision following chronic deprivation amblyopia. Nat Neurosci 10(9):1134-1136. https://doi.org/10.1038/nn1965

Herbison N, Cobb S, Gregson R et al (2013) Interactive binocular treatment (I-BiT) for amblyopia: results of a pilot study of 3D shutter glasses system. Eye (lond) 27(9):1077-1083. https://doi. org/10.1038/eye.2013.113

Hernández-Rodríguez CJ, Piñero D, Molina-Martín A, MoralesQuezada L, de Fez D, Leal-Vega L, Arenillas J, Coco-Martín MB (2020) Stimuli characteristics and psychophysical requirements for visual training in amblyopia: a narrative review. J Clin Med Dec https://doi.org/10.3390/jcm9123985

Hess R, Thompson B (2013) New insights into amblyopia: binocular therapy and non-invasive brain stimulation. J AAPOS 17(1):8993. https://doi.org/10.1016/j.jaapos.2012.10.018

Hess R, Mansouri B, Thompson B (2011) Restoration of binocular vision in amblyopia. Strabismus 19(3):110-118. https://doi.org/ 10.3109/09273972.2011.600418

Holopigian K, Blake R, Greenwald M (1986) Selective losses in binocular vision in anisometropic amblyopes. Vis Res 26(4):621630. https://doi.org/10.1016/0042-6989(86)90010-6

Huang C, Zhou Y, Lu Z (2008) Broad bandwidth of perceptual learning in the visual system of adults with anisometropic amblyopia. Proc Natl Acad Sci U S A 105(10):4068-4073. https://doi.org/ 10.1073/pnas.0800824105

Huang C, Zhou J, Lu Z, Zhou Y (2011) Deficient binocular combination reveals mechanisms of anisometropic amblyopia: Signal attenuation and interocular inhibition. J vis. https://doi.org/10. 1167/11.6.4410.1167/11.6.4

Julesz B, Miller J (1975) Independent spatial-frequency-tuned channels in binocular fusion and rivalry. Perception 4(2):125-143. https://doi.org/10.1068/p040125
Kanski J, Bowling B (2016) Kanski, oftalmología clínica, 8th edn. Elsevier España, Barcelona

Levi DM, Li RW (2009) Perceptual learning as a potential treatment for amblyopia: a mini-review. Vis Res 49(21):2535-2549. https://doi.org/10.1016/j.visres.2009.02.010

Levi D, Knill D, Bavelier D (2015) Stereopsis and amblyopia: a mini-review. Vis Res 114:17-30. https://doi.org/10.1016/j.visres.2015.01.002

Li RW, Provost A, Levi DM (2007) Extended perceptual learning results in substantial recovery of positional acuity and visual acuity in juvenile amblyopia. Invest Ophthalmol vis Sci 48(11):5046-5051. https://doi.org/10.1167/iovs.07-0324

Li J, Thompson B, Deng D, Chan L, Yu M, Hess R (2013) Dichoptic training enables the adult amblyopic brain to learn. Curr Biol 23(8):R308-R309. https://doi.org/10.1016/j.cub.2013.01.059

Mansouri B, Singh P, Globa A, Pearson P (2014) Binocular training reduces amblyopic visual acuity impairment. Strabismus 22(1):1-6. https://doi.org/10.3109/09273972.2013.877945

Mataga N, Mizuguchi Y, Hensch TK (2004) Experience-dependent pruning of dendritic spines in visual cortex by tissue plasminogen activator. Neuron 44(6):1031-1041. https://doi.org/10. 1016/j.neuron.2004.11.028

McColl S, Ziegler L, Hess R (2000) Stereodeficient subjects demonstrate non-linear stereopsis. Vis Res 40(9):1167-1177. https:// doi.org/10.1016/s0042-6989(00)00025-0

McKee S, Levi D, Movshon J (2003) The pattern of visual deficits in amblyopia. J vis 3(5):380-405. https://doi.org/10.1167/3.5.5

Meier K, Giaschi D (2017) Unilateral amblyopia affects two eyes: fellow eye deficits in amblyopia. Invest Ophthalmol vis Sci 58(3):1779-1800. https://doi.org/10.1167/iovs.16-20964

Merchante Alcántara MM (2013) Estrabismo y Ambliopía. Pediatría Integral XVII(7):489-506

Mitchell D, Kind P, Sengpiel F, Murphy K (2003) Brief daily periods of binocular vision prevent deprivation-induced acuity loss. Curr Biol 13(19):1704-1708. https://doi.org/10.1016/j. cub.2003.09.026

Moret B, Camilleri R, Pavan A, LoGiudice G, Veronese A, Rizzo R, Campana G (2018) Differential effects of high-frequency transcranial random noise stimulation (hf-tRNS) on contrast sensitivity and visual acuity when combined with a short perceptual training in adults with amblyopia. Neuropsychologia 114:125-133. https://doi.org/10.1016/j.neuropsychologia.2018. 04.017

Nabie R, Andalib D, Khojasteh H, Aslanzadeh S (2019) Comparison of the effect of different types of experimental anisometropia on stereopsis measured with titmus, randot and TNO stereotests. J Ophthal vis Res 14(1):48-51. https://doi.org/10.4103/jovr.jovr_ 189_17

Ogle K, Wakefield J (1967) Stereoscopic depth and binocular rivalry. Vis Res 7(1-2):89-98. https://doi.org/10.1016/0042-6989(67) 90029-6

Oray S, Majewska A, Sur M (2004) Dendritic spine dynamics are regulated by monocular deprivation and extracellular matrix degradation. Neuron 44(6):1021-1030. https://doi.org/10.1016/j.neuron. 2004.12.001

Paudel N (2018) Smartphone applications for amblyopia treatment: a review of current apps and professional involvement. Telemed J E Health 24(10):797-802. https://doi.org/10.1089/tmj.2017.0220

Polat U (1999) Functional architecture of long-range perceptual interactions. Spat vis 12(2):143-162. https://doi.org/10.1163/15685 6899x00094

Polat U (2009) Perceptual learning induces neuroplasticity, enabling improvement of visual functions. Expert Rev Ophthlamol 4(6):573-576. https://doi.org/10.1586/eop.09.54 
Polat U, Ma-Naim T, Belkin M, Sagi D (2004) Improving vision in adult amblyopia by perceptual learning. Proc Natl Acad Sci USA 101(17):6692-6697. https://doi.org/10.1073/pnas.0401200101

Robaei D, Huynh S, Kifley A, Gole G, Mitchell P (2007) Stereoacuity and ocular associations at age 12 years: Findings from a population-based study. J AAPOS 11(4):356-361. https://doi.org/10. 1016/j.jaapos.2006.11.111

Saraiva AA, Barros MP, Nogueira AT, Fonseca-Ferreira NM, Valente A (2018) Virtual interactive environment for low-cost treatment of mechanical strabismus and amblyopia. Information 9(7):175. https://doi.org/10.3390/info9070175

Smith S, Trachtenberg J (2007) Experience-dependent binocular competition in the visual cortex begins at eye opening. Nat Neurosci 10(3):370-375. https://doi.org/10.1038/nn1844

Spolidoro M, Sale A, Berardi N, Maffei L (2009) Plasticity in the adult brain: lessons from the visual system. Exp Brain Res 192(3):335341. https://doi.org/10.1007/s00221-008-1509-3

Varadharajan S, Hussaindeen J (2012) Visual acuity deficits in the fellow eyes of children with unilateral amblyopia. J AAAPOS 16(1):41-45. https://doi.org/10.1016/j.jaapos.2011.09.016

Vedamurthy I, Knill DC, Huang SJ, Yung A, Ding J, Kwon OS, Bavelier D, Levi DM (2016) Recovering stereo vision by squashing virtual bugs in a virtual reality environment. Phil Trans Soc B 371(1697):20150264. https://doi.org/10.1098/rstb.2015.0264 von Noorden GK (1981) New clinical aspects of stimulus deprivation amblyopia. Am J Ophthalmol 92(3):416-421. https://doi.org/10. 1016/0002-9394(81)90534-1

von Noorden GK Campos EC (2002) Binocular Vision and Ocular Motility, Theory and Management of Strabismus. St Louis, Mosby

Waddingham PE, Butler TK, Cobb SV et al (2006) Preliminary results from the use of the novel Interactive binocular treatment (I-BiT) system, in the treatment of strabismic and anisometropic amblyopia. Eye (lond) 20(3):375-378. https://doi.org/10.1038/sj.eye. 6701883

Zhou Y, Huang C, Xu P, Tao L, Qiu Z, Li X, Lu Z (2006) Perceptual learning improves contrast sensitivity and visual acuity in adults with anisometropic amblyopia. Vis Res 46(5):739-750. https:// doi.org/10.1016/j.visres.2005.07.031

Žiak P, Holm A, Halička J, Mojžiš P, Piñero DP (2017) Amblyopia treatment of adults with dichoptic training using the virtual reality oculus rift head mounted display: preliminary results. BMC Ophthalmol 17(1):105. https://doi.org/10.1186/s12886-017-0501-8

Publisher's Note Springer Nature remains neutral with regard to jurisdictional claims in published maps and institutional affiliations. 